\title{
Web-services Based Conversational Agent to Encourage Willingness to Communicate in the EFL Context
}

\author{
Emmanuel Ayedoun*, Yuki Hayashi* and Kazuhisa Seta*
}

(Received 15 June 2015 and accepted in revised form 30 May 2016)

\begin{abstract}
In this paper, we propose an embodied conversational agent based on the willingness to communicate (WTC) model in L2 to help increase WTC in the context of English as a foreign language (EFL) by providing users with various daily conversation contexts. To simulate realistic and efficient conversations, we adopted a semantic approach in the response generation and created a system with flexible and adaptable domain knowledge, user's intent detection, and mixed-initiative conversation strategy. Our evaluation of the proposed system demonstrated its potential to simulate efficiently natural conversations in a specific context as well as the feasibility of improving learners' WTC using a computer-based environment.
\end{abstract}

Keywords: willingness to communicate, web-services integration, second language acquisition, embodied conversational agent, intelligent tutoring

\section{Introduction}

One of the primary goals of second language (L2) and foreign language learning is to facilitate better communication and understanding between individuals who come from different cultural backgrounds and speak different languages ${ }^{(1)}$. However, even after studying for several years, many L2 learners will not spontaneously be willing to engage in conversation in their L2. For example, in Japan, although more attention is being given to speaking by emphasizing the importance of communicative skills in English, most university students cannot speak English adequately, even after studying it for six years in secondary school.

MacIntyre et al. ${ }^{(2)}$ suggested that the key factor predicting frequency of L2 use is the willingness to communicate (WTC) in L2, defined as a "readiness to enter into discourse at a particular time with a specific person or persons, using an L2". They proposed a heuristic model of variables influencing WTC in which situated antecedents (desire to communicate with a specific person and state communicative self-confidence) have a direct and substantial impact on a learner's decision to participate (or not) in an L2 conversation. Assuming that those situated antecedents are seen as transient and dependent on the specific context in which a person functions at a given time, it becomes obvious that the

*Graduate School of Humanities and Sustainable System

Sciences, Osaka Prefecture University, Japan learning environment plays an important role in motivating the learner to communicate in L2.

In this paper, we propose a computer-based conversational environment built to enhance WTC in English by providing learners with opportunities to naturally simulate daily conversations in various social contexts $^{(3)}$. Following the new architectural trends in service driven computing, our approach aims to build a dynamic and adaptable system by combining several existing web-services in order to fulfil the multiple requests related to natural language processing and the constraints that have to be taken into consideration in order to improve a learner's WTC.

\section{Related Works}

Linguistic proficiency and the ability to use a language in communicative situations are two of the primary goals of L2 learning. Researchers have found that use of the target language plays a crucial role in L2 acquisi$\operatorname{tion}^{(4)}$. Others have also emphasized the role of output in L2 learning, arguing that output is necessary for the development of production (talking and writing), as input develops only listening and reading comprehension ${ }^{(5,6)}$. However, a pressing issue in L2 learning is how to encourage learners to use L2 for communication. It is interesting that, despite excellent linguistic competence, some students avoid using L2 for communication, whereas other students with only minimal linguistic competence seem to communicate in the L2 whenever possible. 


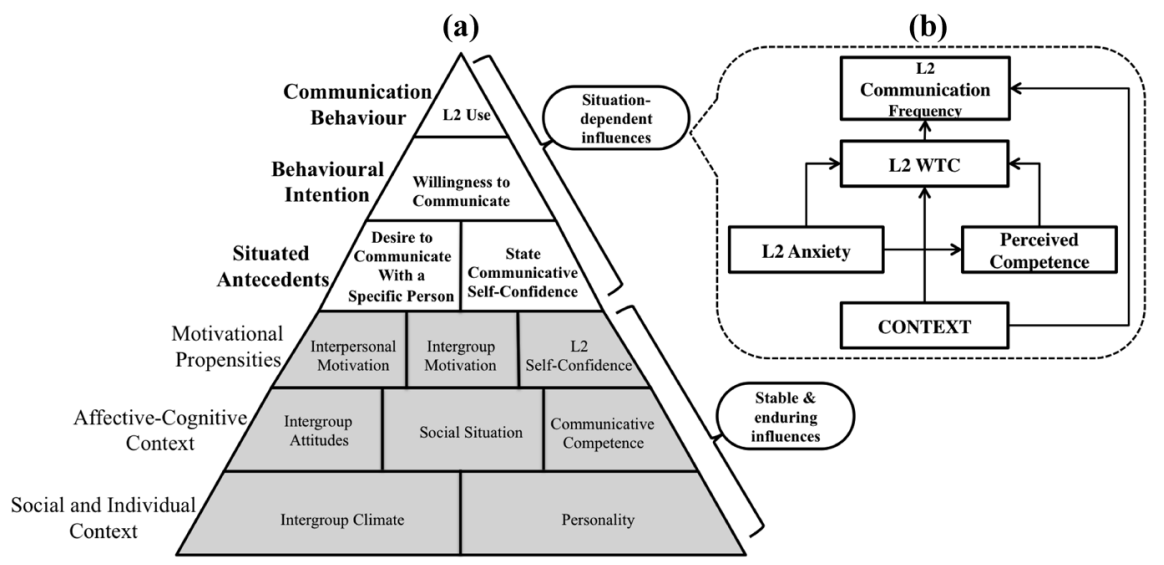

Figure 1. (a) Heuristic Model of Variables Influencing WTC ${ }^{(2)}$; (b) A Portion of the L2 WTC Path Model ${ }^{(14)}$.

MacIntyre et al. ${ }^{(2)}$ explained such differences by the fact that output is not directly affected by linguistic competence. Rather, they identified WTC as the key factor to ensure a spontaneous and sustained use of the L2 and suggested that increasing WTC should be the goal of L2 learning. They proposed a pyramidal heuristic model of variables affecting WTC (Figure 1(a)), consisting of six layers: the top half (communication behaviour, behavioural intention, situated antecedents) represents situation-dependent influences on WTC at a given moment in time and the bottom half (motivational propensities, affective-cognitive context, social and individual context) describes stable, enduring influences on the process.

We assume that both an immersive social context and a great amount of output are necessary to enable learners to gain satisfaction and self-confidence and lower their anxiety, thereby increasing their WTC in L2. As mentioned above, situated antecedents are believed to affect WTC. Baker and MacIntyre ${ }^{(7)}$ examined the differences in nonlinguistic outcomes of French immersion versus non-immersion programs. As expected, immersion students displayed higher WTC and more frequent communication in French. Yashima et al. ${ }^{(1)}$ investigated WTC among Japanese high school students who stayed temporarily in the United States. They found that those who communicated with hosts more frequently seemed to have had a higher degree of satisfaction in human relationships, experienced less difficulty in making friends, and perceived their adjustment to the host country to be better than those who engaged in communica- tion less frequently. However, most L2 learners have few opportunities to access such immersive social contexts. Isoda $^{(8)}$ mentioned that many Japanese university students hesitate to talk because they have little experience speaking English in Japan. Increasing learners' WTC in L2 is a problem that is difficult to overcome due to the lack of suitable conversation environments.

Several studies have suggested that computerbased learning environments have the potential to be an efficient alternative to real interactions. Nass et al. ${ }^{(9)}$ conducted several studies on the way people interact with computers and concluded that the human-computer relationship is fundamentally social. Furthermore, Atkinson et al. ${ }^{(10)}$ developed a social agency theory that outlines the effectiveness of animated pedagogical agents (APAs) in human-computer interaction. According to this theory, using verbal and visual cues in a computer-based environment encourages the learners to interpret their interaction with the computer in a partnership. Learners consider their interaction with the computer as a social one because the social cues are similar to what they would expect from a human-to-human conversation. As discussed by Arslan-Ari ${ }^{(11)}$, the effectiveness of APAs in the educational setting has been studied in various fields, including science, mathematics, and humanities. However, in the field of L2 acquisition, more specifically when considering support for the emotional variables affecting language acquisition, there has been limited research on the application of APAs in multimedia learning environments. Ohmaye ${ }^{(12)}$ suggested that 
multimedia-learning environments with APAs offer L2 learners the opportunity to interact with native speakers and provide a social context. Abu Shawar and Atwell ${ }^{(13)}$ pointed out that Chatbots (programs that pretend to chat with a human user in natural language) appear to be a suitable partner for conversation practice. They used a corpus training approach to train and generate two Chatbots: one called Afrikaana, which speaks Afrikaans, and the other a bilingual version speaking English and Afrikaans, called AVRA. Learners could chat with these Chatbots on any given topic and found it an interesting tool to practice the language. Unfortunately, users also revealed that many responses were not related to the topic or were even nonsense. Errors in the response generation, which is a common issue with Chatbots, might limit the degree of reality of such computer-based conversational environments.

Nakaya and Murota ${ }^{(14)}$ proposed a mobile conversation learning system, which aimed to motivate Japanese EFL learners. In their system, the conversations topics were based on learners' lifelogs or related to situations learners often experience such as restaurant situations. The advantage of such an approach is that it gives learners the possibility to talk about several topics within the same system. On the other side, the number and the order of questions per conversation session are predefined, and the conversation management is mainly system-driven, which means learners cannot ask the system just anything, but they are limited to answering questions generated by the system according to their lifelogs. With such a context-independent conversation management approach, it is really difficult to achieve a high degree of interaction reality since the system does not focus on a particular context.

Although some APAs and conversational agents exist and help simulate real interactions, we could not find any that were specifically suitable to improve learners' WTC in L2.

\section{Building a Conversational Agent}

\subsection{Basic Philosophy}

To improve learners WTC in L2, we focus on the top three layers of the heuristic pyramid of variables affecting $\mathrm{WTC}^{(2)}$. The variables identified in these layers have situation-dependent and immediate influences on WTC and can be stimulated easily compared to other variables in the three bottom layers of the pyramid, which have more stable and enduring influences on WTC but are difficult to elicit because they are related to personality traits or societal norms.

Figure 1(b) shows a portion of the WTC path model in L2 representing causal relations among variables in the top three layers of the pyramid, proposed by MacIntyre and Charos ${ }^{(15)}$. This portion of the model suggests that the intention or willingness to engage in L2 communication is determined by a combination of the perception of learners regarding their second language proficiency (perceived competence), the opportunity to use the language (context), and a lack of apprehension about speaking (L2 anxiety). Indeed, experiencing a particular situation affects both perceived competence and the level of anxiety. This is why unfamiliar situations typically result in a lower WTC while familiar situations in which the interlocutor is free of apprehension positively affect WTC because there is less anxiety felt and a higher perceived competence experienced.

Our system aims to reduce learners' anxiety while improving their self-confidence in English communication by giving them opportunities to simulate and enjoy daily conversations in English. We expect that the continuous use of our system as a scaffolding or practice environment will foster learners' readiness to participate in English conversations in the real world. Such a scaffolding environment is necessary because some L2 learners, when suddenly given the opportunity to speak using the L2 in face-to-face situations, hesitate to talk because they feel ashamed or anxious about the other person's judgment of their linguistic skills. Our system aims to target such learners.

It is very hard to explicitly describe how and why the computer reduces anxiety and increases confidence so we need to carefully address such issues. We hypothesized that if the computer-based conversational environment satisfies requirements such as social conversation contexts, conversation smoothness and learners' immersion, we might be able to reduce learners' anxiety and increase their confidence toward taking the initiative to talk in real situations. Some specifics are given next.

(a) Social conversational contexts: Contexts have a direct influence on WTC in L2, as indicated in Figure 1(b). Since the decision to initiate speech varies consid- 
erably over time and across situations, a desirable conversational environment should offer learners the possibility to converse efficiently in a variety of different situations.

(b) Conversation smoothness: Social context alone is not enough to make a natural and dynamic conversation possible. That is why strategies to keep the conversation going, especially when learners do not feel confident about taking part in the discourse, are desirable: they contribute to creating a friendly conversational environment and reduce the learners' anxiety.

(c) Learners' immersion: The term "immersion" stands here for a sense of "being there" felt by learners when interacting with the system. To help efficiently increase WTC in L2, in contrast to textbooks or "listen and repeat" activities, we assume that the computer-based environment has to be realistic enough to ensure a good immersion for the learners. Adequate immersion in the conversational context is essential because it enables the learners to take part actively in the conversation.

In total, we aim to adopt a context-dependant scenario-based approach to develop a realistic conversational environment with an embodied conversational agent that uses both verbal and non-verbal behaviours in the conversation. This is especially important in order to create an interactive conversational environment where learners can consider the computer-based agent as a partner.

\subsection{System Requirements}

In order to satisfy the requirement related to (a) "social conversational contexts", the system has to deal with a huge amount of knowledge from various contexts. The agent's domain knowledge has to be contextdependent so as to avoid responses that are nonsense or are not related to the current context. In addition, since the domain knowledge is context-dependent, a different context would require a different knowledge model. Therefore, easy editing or replacement of the system's domain knowledge should be implemented in order to make it possible even for those who do not have specific programming skills to adapt the system to various conversation contexts. Also, in order to be credible, the agent has to adopt conversational strategies that are appropriate for human communication. These strategies include the ability to understand learners' utterances as well as the ability to indicate and control the flow of the conversation.

To satisfy requirement (b) "conversation smoothness" requirement, the agent has to be able to adopt conversational functions such as turn-taking. Turn-taking can be seen here as the ability to take the initiative and speak up first when necessary. The advantage to this is that it gives to the agent the ability to recover from errors that might occur during speech recognition by asking for confirmation or making suggestions to the learner.

Although computer-based environments encourage learners to interpret their interaction with the computer as a social one, creating immersive communication environments or reproducing social conversation contexts using computers is still a big challenge. To satisfy requirement (c) "learners' immersion", the conversational agent must provide a high level of performance similar to that for human-to-human conversations in the response generation. A realistic interface, including speech synthesis with an embodied conversational agent that is able to enage in both verbal and non-verbal communication (facial expressions, lip-syncs, etc.), is desirable.

\subsection{System Architecture}

Figure 2 depicts the different modules, both internal (red) and external (purple), that constitute the structure of our conversational agent. The internal modules are composed of the dialogue manager and the multimodal response generator modules. The four external modules are connected to the internal modules and are expected to provide a specific set of functionalities that can be changed or removed according to the situation.

\subsubsection{Dialogue Manager}

The dialogue manager inputs the information it receives from the user's utterance recognition as well as the dialogue script and outputs instructions (utterances, the agent's behaviour, etc.) that are necessary for suitable response generation.

In order to engage learners and maintain productive conversations, adequate management of the conversation state and flow is desirable. By following the dialogue script requirements, our dialogue manager can 


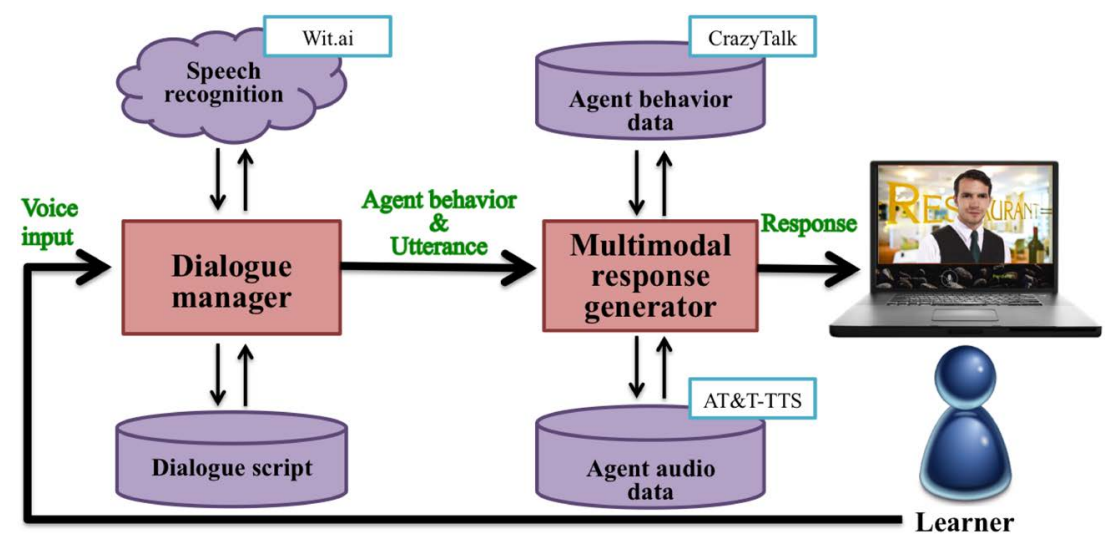

Figure 2. Architecture of the Conversational Agent.

identify which responses or actions are the most suitable to answer learners' utterances and it can also identify when the agent has to take the initiative to lead the conversation. As mentioned above, one of the required abilities for realistic conversational agents is turn taking. In other words, the system should not only wait for and answer learners' utterances but must also be able to take the initiative and talk first when necessary.

Speech recognition: We used Wit.ai ${ }^{(16)}$ because its unique approach to natural speech recognition enables us to turn a learner's utterance into structured data, providing the semantic information ("intent", "entities", etc.) required to implement a semantic approach in the response generation. Because the same intention (e.g. Greeting) can be expressed in multiple ways (e.g. Hey, Good morning, Hello), being able to understand what the learner has said ("intent") is the first step to enable semantic processing of the learner's utterance by the conversational agent. It also gives our system the ability to handle various utterances that learners use to convey the same meaning. Moreover, Wit.ai provides a simple platform where the knowledge base of our conversational agent can be easily constructed and organized in conversation instances according to the topic or target context. Basically, all the intents needed for a particular conversation must be declared on Wit.ai so that they can be identified later from learner's utterances. If the intents are properly defined, there is no limit to the number of intents that the system can handle. That unique feature makes our agent adaptive and flexible enough to meet the different context requirements. Another advantage of Wit.ai is that it generates confidence ratings for each response, enabling us to adjust the conversation or even fix recognition or interpretation errors for more convincing interactions with the learners.

Dialogue script: As mentioned above, WTC is highly influenced by contextual factors. Since each conversation context has unique characteristics, each requires a specific dialogue script. Figure 3 shows an example portion of the dialogue script structure in the current system. We opted for a structured data style of dialogue script, written in XML so that users can easily modify, extend, or add new conversation contexts to the system. Information that helps manage the conversation such as utterance scripts, agent behaviours, waiting time, and next content are organized in the dialogue script. Thus, in order to implement a new conversation in the system, we only need to replace the dialogue script, without having to modify the system's main components. That is to say by just editing or replacing the dialogue script, our system can be used to manage different conversations situations.

To let the system be aware of the conversation flow and modify it accordingly to the detected utterance, we implemented the concept of steps in the dialogue script. By steps here we mean a specific stage in the conversation flow, and a given conversation can be split into several steps. For example, given "Talk with a waiter at a restaurant" as the conversation context, the following basic steps can be imagined: Enter restaurant - Get seat 


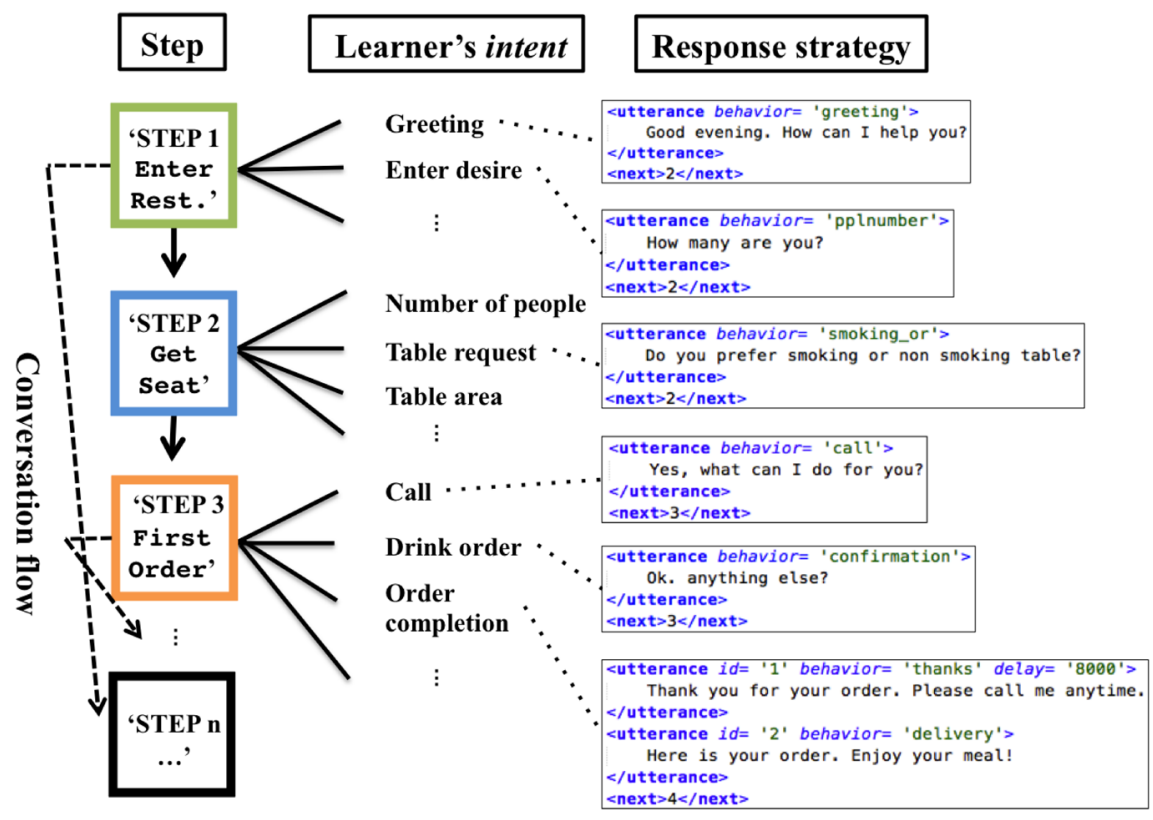

Figure 3. Example of Dialogue Script Structure.

- Order - Drink/Eat - Settle bill. In our system, when an intent (e.g., Greeting) is identified from the learner's utterance (e.g., "Good evening"), the corresponding utterance script for output (e.g., "Good evening. How can I help you?") is identified by referring to the conversation's current step (e.g., 'STEP 1: Enter Rest.'). This also enables the system to deal more efficiently with ambiguity, which is a major challenge when building conversational agents. In case an undefined intent is detected from the learner's utterance, our agent can ask confirmation or make suggestions to the learner according to the dialogue script in order to make the conversation move forward.

Combining a learner's utterance (intent) with contextual information (step) enables us to go beyond simple word matching and semantically analyze the learner's utterance for better response generation and avoidance of nonsense answers. Furthermore, using steps makes turn taking easier for the agent, since it is aware of the current stage of the conversation and the agenda that has to be fulfilled in order to clear that stage. Moreover, using steps brings more flexibility to the dialogue flow because its clearance order may change in accordance with a learner's utterance or con- text-dependant local constraints such as time limitations and opening hours for example. An example showing flexible and adaptive dialogue management is given in Section 3.4.

\subsubsection{Multimodal Response Generator}

The set of instructions obtained from the dialogue manager is executed and displayed on the user interface by the response processor module. These instructions are related to verbal (utterances) and non-verbal (facial expressions, head movements, eye gaze, etc.) behaviours that are performed by the agent in order to converse with the learner.

Agent audio data: In order to increase the naturalness of the conversation, our agent has to generate high quality output. The utterances obtained from the dialog script are turned into audio files using AT\&T-TTS ${ }^{(17)}$, which provides us with a wide range of natural voices that can be modified according to need. It is possible to control the way the speech sounds in order to generate better phrasing or even to control the overall speed by using XML-style tags. 
Agent behaviour data: Our conversational agent's interface character is built on CrazyTalk ${ }^{(18)}$ using Unity technology. This is a facial animation tool that enables us to produce non-verbal output such as vivid facial expressions as well as lip-syncs in fine detail. According to the context or scenarios, different types of characters (photo-based or CG-rendered actors, or illustrative style characters) can be easily chosen to provide learners with a realistic interface. In addition, the interactive plug-in functionality of CrazyTalk lets us easily incorporate our conversational agent into Web pages, which increases the portability of the system.

\subsection{Prototype System}

We built a prototype of the system described above simulating the context of a conversation held in a restaurant. The user interface is accessible via a webbrowser and mainly displays the agent behaviours during the conversation. Below the agent behaviours frame, there is a microphone icon on which learners can click to input their utterance. Agent's utterances are also displayed on the screen to facilitate better understanding for learners. In this prototype system, the dialogue script used is structured as five steps composed of 18 conceivable intents in the restaurant context.

Figure 4 shows screen images of our system, where a conversational agent plays the role of waiter while the learner interacts with the system playing the role of the customer. Figure 5 shows an excerpt from a sample conversation. Intent detected from the learner's utterance is shown in italics in brackets. Steps are also marked to facilitate better understanding of the conversation strategy. This conversation gives us an overview about how our agent can recover from errors that may occur during the speech recognition phase. Actually, in 'STEP 3 First Order', the second utterance of the learner was recognized as "Deer please" instead of "Beer please". As a result, the detected intent was "undefined" since "Deer" does not refer to any drink or food in this context. To recover from that error, here the agent asks for confirmation to the learner about the precedent utterance. Hopefully, the second time, the learner's intent is accurately detected and the conversation can move forward.

Figure 6 shows excerpts of two different conversations occurring in the current prototype system. It gives an overview of how our system controls the dialogue flow accordingly to the learner's utterances and con-

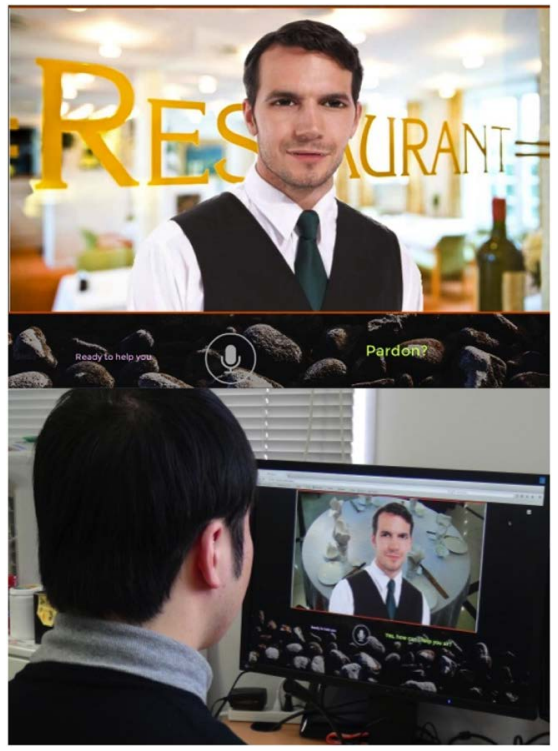

Figure 4. User Interacting with the Agent.

Agent: Welcome to xxx restaurant.

Learner: Hello! (Greeting)

Agent: Good evening. How can I help you?

'STEP 1

Enter Rest.'

Learner: A table for two please. (Table request)

Agent: Do you prefer smoking or

Learner: Non-smoking table (Table area)

Agent: All right, please come this way. (Wait for 3 seconds)

'STEP 2

Get Seat'

Here is your table, please call me when you are ready to order.

Learner: Thank you. (Thanks)

Agent: You are welcome.

Learner: Deer please. (Undefined)

Agent: Pardon?

Learner: Beer. (Drink order)

Agent: Ok. Anything else?

Figure 5. Conversation Example.

straints related to the conversation context. In the conversation excerpt on the left, the dialogue flow evolves from 'STEP 3 First Order' to 'STEP 4 Last Order' because the local constraint $<$ Time of the first order has elapsed $>$ was fulfilled. From 'STEP 4 Last Order', the 


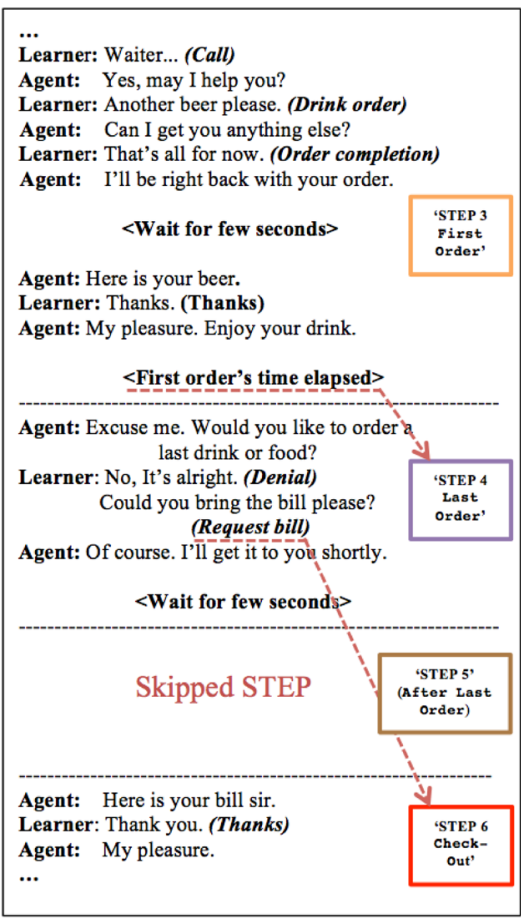

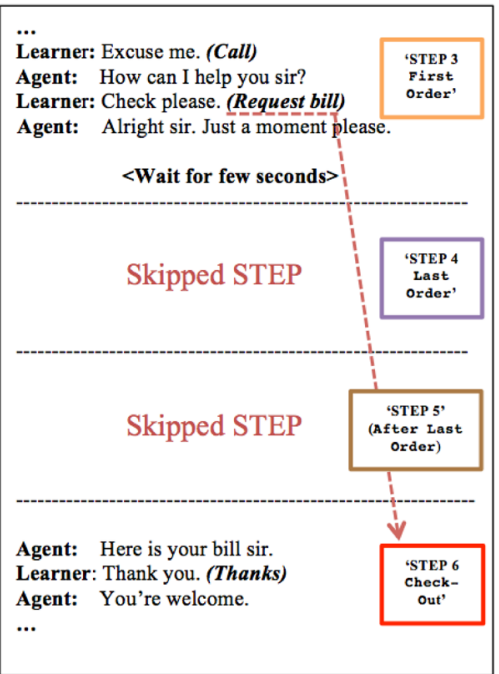

Figure 6. Flexible Dialogue Flow Management. conversation moves directly to 'STEP 6 Check Out' because a specific intent (Request bill) was detected from the learner's utterance ("Could you bring the bill please?"). On the other side, in the conversation excerpt on the right, the dialogue flow is different. From 'STEP 3 First Order', the conversation evolves directly to 'STEP 6 Check out' without going through 'STEP 4 Last Order' or 'STEP 5 After Last Order' because a specific intent (Request bill) was detected from the learner's utterance ("Check please"). These examples illustrate how according to the detected intent from the learner's utterances and context-dependant local constraints, our system is able to realize flexible control of dialogue flow in a restaurant context.

\section{System Evaluation}

We conducted a set of experiments to evaluate the feasibility of simulating natural interactions with our system and a preliminary study to appraise the effects of our prototype system on WTC in the context of English as a foreign language (EFL). In the preliminary study, we analysed the differences between participants' WTC before and after their interaction with our system. Also, we evaluated the reality degree of conversation contexts provided by the system itself. We assumed that the results would be viewed as positive if our system was able not only to reduce learners' anxiety while improving their self-confidence but also to provide an immersive conversational environment in which learners could feel a certain amount of pressure similar to what they would feel during real conversations. This would validate the degree of reality provided by this system.

Prior to the study, first we had to evaluate the feasibility of simulating natural interactions with our system (whether or not our agent was able to carry on natural conversations with users). To this end, we asked two students to interact with the system and we recorded their interactions after obtaining their agreement. In order to keep the most natural environment possible for the learners, we chose to record the interactions rather than staying in the room and observing them directly. 
From the recorded interactions, we could observe that our agent was able to converse successfully with users. Even when users remained silent or could not find appropriate words to express themselves, our agent, following the dialogue script, adopted a positive conversation strategy by asking confirmation or making suggestions to learners in order to keep the conversation going. Thus, we concluded that the performance of our system was satisfying enough in order to be used for the evaluation study.

\subsection{Procedures and Materials}

For this pilot study, the participants were five Japanese undergraduate or graduate students currently attending a Japanese university. They were asked to fill in some questionnaires and to converse with our conversational agent. All participants were given as much time as required to complete the questionnaires. Data were collected anonymously and participants were told that it would be kept confidential. The experimental study was conducted in accordance with the following three procedures.

Procedure 1 (Measures of WTC): We used a survey developed by Matsuoka ${ }^{(19)}$ and inspired from the WTC test of Sick and Nagasaka ${ }^{(20)}$ to gather data about WTC before and after interactions with our conversational agent.

The WTC survey taken before interaction with the system consisted of three variables. Participants were asked to rate 30 scenarios (e.g., Making a telephone call in order to make a reservation at a hotel in an English speaking country) related to using English in various circumstances with a four-point Likert scale. As described in Table 1, the first variable tests for confidence and asks participants to rate the scenarios from 0 (I absolutely don't think I could do that) to 3 (I think I could do that easily). The second variable tests for nervousness and asks participants to rate the same scenarios from 0 ( $I$ would definitely not be nervous) to 3 ( $I$ would be extremely nervous). Finally, participants were asked to rate the third variable, desire to communicate in English from 0 (I wouldn't want to try) to 3 (I would absolutely want to try that).

The WTC survey used after interactions with our conversational agent was similar to the survey described above but differed in that participants were asked to rate
Table 1. Rates Scale of Variables' Table Used for Procedure 1.

\begin{tabular}{|c|c|c|c|}
\hline & Confidence & Nervousness & Desire \\
\hline 3 & $\begin{array}{l}\text { I think I could do } \\
\text { that easily. }\end{array}$ & $\begin{array}{l}\text { I would be } \\
\text { extremely } \\
\text { nervous. }\end{array}$ & $\begin{array}{l}\text { I would absolutely } \\
\text { want to try that. }\end{array}$ \\
\hline 2 & $\begin{array}{l}\text { I think I could do } \\
\text { that. }\end{array}$ & $\begin{array}{l}\text { I would be } \\
\text { nervous. }\end{array}$ & $\begin{array}{l}\text { I would want to } \\
\text { give it a try if } \\
\text { given the chance. }\end{array}$ \\
\hline 1 & $\begin{array}{l}\text { I don't think } \\
\text { I could that. }\end{array}$ & $\begin{array}{l}\text { I would be } \\
\text { a little nervous. }\end{array}$ & $\begin{array}{l}\text { It depends on the } \\
\text { situation. }\end{array}$ \\
\hline $\mathbf{0}$ & $\begin{array}{l}\text { I absolutely } \\
\text { don't think } \\
\text { I could do that. }\end{array}$ & $\begin{array}{l}\text { I would definitely } \\
\text { not be nervous. }\end{array}$ & $\begin{array}{l}\text { I wouldn't want } \\
\text { to try that. }\end{array}$ \\
\hline
\end{tabular}

Table 2. Rates Scale of Variables' Table Used for Procedure 2.

\begin{tabular}{|c|c|c|c|}
\hline & Confidence & Nervousness & Desire \\
\hline 3 & $\begin{array}{l}\text { I think I'd } \\
\text { become able to } \\
\text { do that easily. }\end{array}$ & $\begin{array}{l}\text { I think I'd be } \\
\text { extremely } \\
\text { nervous. }\end{array}$ & $\begin{array}{l}\text { I think I'd abso- } \\
\text { lutely be willing } \\
\text { to try that }\end{array}$ \\
\hline 2 & $\begin{array}{l}\text { I think I'd } \\
\text { become able to } \\
\text { do that. }\end{array}$ & $\begin{array}{l}\text { I think I'd be } \\
\text { nervous. }\end{array}$ & $\begin{array}{l}\text { I think I'd be } \\
\text { willing to try } \\
\text { given the chance. }\end{array}$ \\
\hline 1 & $\begin{array}{l}\text { I don't think I'd } \\
\text { become able to } \\
\text { do that. }\end{array}$ & $\begin{array}{l}\text { I think I'd be a } \\
\text { little nervous. }\end{array}$ & $\begin{array}{l}\text { It depends on the } \\
\text { situation. }\end{array}$ \\
\hline $\mathbf{0}$ & $\begin{array}{l}\text { I absolutely } \\
\text { don't think I'd } \\
\text { become able to } \\
\text { do that. }\end{array}$ & $\begin{array}{l}\text { I think I won't be } \\
\text { nervous. }\end{array}$ & $\begin{array}{l}\text { I don't think I'd } \\
\text { feel like trying }\end{array}$ \\
\hline
\end{tabular}

the same scenarios imagining how high their confidence, anxiety, and desire would be if they were able to frequently interact with our conversational agent. In the first section of the test, they were asked to evaluate their confidence from 0 (I absolutely don't think I'd become able to do that) to 3 (I think I'd become able to do that easily). In the second section, which tests for nervousness, they were asked to rate from 0 (I think I won't be nervous) to 3 (I think I'd be extremely nervous). As described in Table 2, in the third section, they rated their desire to communicate in English from 0 (I don't think I'd feel like trying) to 3 (I think I'd absolutely be willing to try that).

Procedure 2 (Interaction with system): After filling 
out the first WTC survey, participants were asked to converse with the agent as much as they wanted for 15 minutes. They were advised to simulate a conversation in a restaurant with them playing the role of customer. In order to let them focus and converse with the agent in the most possible natural environment without any fear or anxiety about people who might be hearing or staring at them, each participant was left alone in a room specially prepared for the experiment. They were also informed that their conversation with the agent would not be monitored or recorded, giving them the assurance that they could relax and enjoy the interactions like they would do during real interactions.

Procedure 3 (Measure of reality degree): In order to evaluate the quality and the naturalness of the conversational environment provided by our system, participants were asked to rate three items (nervousness during interaction, degree of immersion, fluency of conversation) with a five-point Likert scale. They were first asked to rate their degree of nervousness during the interaction from 0 (I wasn't nervous at all) to 4 (I felt nervous like in a real conversation), then the degree of immersion from 0 (I wasn't immersed at all) to 4 (I felt like I was having a real conversation), and finally the fluency of their interaction with the system from 0 (It didn't go well at all) to 4 (The conversation went smoothly).

\subsection{Results}

Figure 7 shows the results from the two WTC tests administered before and after interacting with our conversational agent. As shown, after interacting with the agent, the learner's confidence and desire for communication slightly increased (by +0.4 and +0.1 , respectively) while their nervousness decreased (by 0.2 ). We analyzed the results in detail and found that after interacting with the system, $80 \%$ of examinees declared they were more confident and more desirous of interacting in English than before. On the basis of these results, we can say that our system does not have a negative impact on learners' WTC and we can also predict that a continuous usage of the current system might increase both user desire and self-confidence in terms of communication in English. However, at this point, due to the limited number of examinees (5), we could not make any statistical analysis to prove the positiveness of the

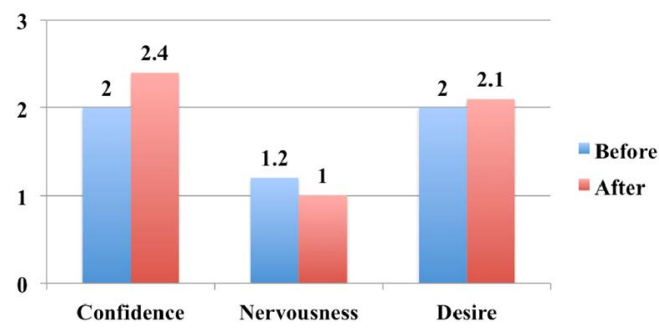

Figure 7. WTC Variables before and after Interactions.

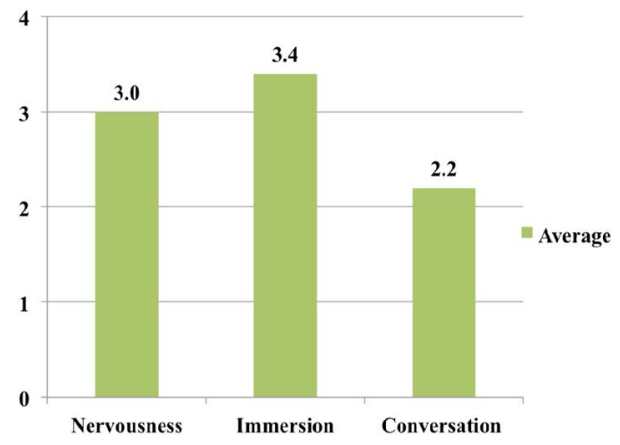

Figure 8. Perception of Interaction's Degree of Reality.

results obtained.

Figure 8 shows the degree of reality of the system according to the questionnaire results. As showed, learners felt nervous (the same as in real conversations) during their conversations. Experiencing some nervousness during public speaking is normal, so the high nervousness rate observed during the interaction (3.0) is positive in terms of the conversation naturalness provided by the system. Another reason to explain the high nervousness rate could be the fact that learners did not feel confortable conversing with a computer as a partner. However, in that case, the immersion level should have been low but in our case, the immersion level was good (3.4) supporting the naturalness of the interactions as mentioned above. Conversation fluency was acceptable (2.2).

\subsection{Discussion and Limitations}

About the system itself, these results suggest that even though the conversation fluency was not very high, the conversational environment provided by our system is realistic enough to enable a good immersion of learn- 
ers with suitable pressure (without high anxiety) in the conversational context. Thus, our system is able to provide an environment to enable learners to simulate natural conversations, which validates our approach to combine several existing web-services to build a more realistic agent.

As far as the contribution to learners' WTC is concerned, assuming that context, self-confidence and low anxiety have a strong and direct influence on WTC in EFL (Figure 1(b)), the obtained results suggest that interactions with our conversational agent might have a positive effect on the learners' WTC.

We got both positive and negative feedbacks from learners after they interacted with the system. Regarding the usefulness of the system, most of the learners found the system useful because it gave them an opportunity to simulate conversation situations similar to what they might experience with native English speakers in the real world. Regarding the scalability, they suggested that we should implement more conversational contexts so that individual learners could freely choose which situation to practice according to their preferences. Regarding the conversation smoothness, a few of the users pointed out some difficulties related to the fact that sometimes their utterances could not be understood by the system, which limited the smoothness of the interactions.

However, when we consider that the conversational environment provided by our prototype system is limited to conversation in a public context (a restaurant), better results would certainly be obtained if we add more conversational contexts to the system. We will continue to increase the domain knowledge of the current conversational agent in order to provide the opportunity for conversations in many social contexts. Also, since learners' WTC, of course, do not increase overnight, we understand that a certain amount of continuous usage of the current system with the possibility for learners to converse in various contexts is necessary before we can collect more reliable data. Therefore, although the results obtained in this pilot study are promising, they should be regarded as tendencies that have to be confirmed with more complete evaluations.

\section{Conclusion}

In this paper, we presented a conversational agent to increase learners' WTC in the EFL context.
Yashima ${ }^{(21)}$, for instance identified the "International Posture" as a specific factor influencing WTC in the Japanese EFL context. The objective of this research is not limited to the Japanese context but is ideally to propose a more or less general and efficient computerbased model to enhance WTC in any EFL context. That is the reason why the system concept was built on the WTC path model developed by MacIntyre and Charos $^{(15)}$, which is commonly seen as a basic and universal reference model of key factors influencing WTC in $\mathrm{L} 2$ acquisition. Our conversational agent was built by successfully integrating several existing web-services rather than creating an agent from scratch for each instance, which would have needed a great amount of time and resources. Moreover, this architecture makes our agent flexible and adaptable enough to be used in various conversation contexts.

Our evaluation showed that the conversational environment provided by the system might have a positive influence on the key factors predicting WTC in L2 and suggests that frequent usage of the system can increase learners' self-confidence and desire to communicate in English in daily conversation contexts. In addition, the degree of reality of the system environment was enough to enable a good immersion of learners in the conversation context.

In future work, we will add more conversation contexts to the current prototype system in order to provide users with a richer pool of conversational contents. To this end, we will identify which conversational contexts our system should provide in order to be more efficient. To adapt our system to specific contexts such as Japanese, Chinese or Turkish for example, we will certainly have to take into consideration additional factors influencing WTC that are proper to each of those contexts (e.g. the "International Posture" in the Japanese EFL context). We also intend to focus on the interface design and provide the conversational agent with more communicative features in order to enable a better immersion of users in the conversation context. Finally, larger scale experiments should be conducted to verify the tendencies reported in this paper and to clarify the degree to which the system encourages WTC in the EFL context.

\section{References}

(1) Yashima, T., Zenuk-Nishide, L. and Shimizu, K.: "The 
Influence of Attitudes and Affect on Willingness to Communicate and Second Language Communication", Language Learning, Vol. 54, No. 1, pp. 119-152 (2004).

(2) MacIntyre, P. D., Clément, R., Dörnyei, Z. et al.: "Conceptualizing Willingness to Communicate in a L2: A Situational Model of L2 Confidence and Affiliation", Modern Language J., Vol. 82, pp. 545-562 (1998)

(3) Ayedoun, E., Hayashi, Y. and Seta, K.: “A Conversational Agent to Encourage Willingness to Communicate in the Context of English as a Foreign Language", Proc. of the19th International Conference on Knowledge Based and Intelligent Information and Engineering Systems, Vol. 60, pp. 1433-1442 (2015).

(4) Seliger, H. W.: "Does practice make perfect?: A Study of Interaction Patterns and L2 Competence", Language Learning, Vol. 27, pp. 263-278 (1977).

(5) Swain, M. and Lapkin, S.: "Problems in Output and the Cognitive Processes they Generate: A Step towards Second Language Learning", Applied Linguistics, Vol. 16, No. 3, pp. 371-391 (1995).

(6) Swain, M.: "Focus on Form through Conscious Reflection", in Focus on Form in Classroom Second Language Acquisition, eds. Doughty, C. and Williams, J., pp. 64-81. Cambridge University Press, Cambridge (1998).

(7) Baker, S. and MacIntyre, P. D.: "The Role of Gender and Immersion in Communication and Second Language Orientations", Language Learning, Vol. 50, pp. 311-341 (2000).

(8) Isoda, T.: "Developing a Scale of Unwillingness to Speak English", Hiroshima Studies in Language and Language Education, Vol. 11, pp. 41-49 (2008).

(9) Nass, C.I., Steuer, J. S. and Tauber, E.: "Computers Are Social Actors", Proc. of the CHI '94, pp. 72-78 (1994).

(10) Atkinson, R.K., Mayer, E. M. and Merill, M. M.: "Fostering Social Agency in Multimedia Learning: Examining the Impact of an Animated Agents Voice", Contemporary Educational Psychology, Vol. 30, pp. 117139 (2005).

(11) Arslan-Ari, I.: "Animated Pedagogical Agents from
Students' Perspectives", Proc. of the Annual Convention of the Association for Educational Communications and Technology, Vol. 1, pp. 6-9 (2010).

(12) Ohmaye, E.: "Simulation-based Language Learning: An Architecture and a Multi-media Authoring Tool", in Inside Multi-media Case Based Instruction, ed. Schank, R. C., pp. 1-102. L. Erlbaum Associates, Mahwah, N.J. (1998).

(13) Abu Shawar, B. and Atwell, E.: "Fostering Language Learner Autonomy through Adaptive Conversation", Proc. of the Corpus Linguistics Conference, CL'2007 (2007).

(14) Nakaya, K. and Murota, M.: "Development and Evaluation of an Interactive English Conversation Learning System with a Mobile Device Using Topics Based on the Life of the Learner", Research and Practice in Technology Enhanced Learning, Vol. 18, No. 1, pp. 65-89 (2013).

(15) MacIntyre, P. D. and Charos, C.: "Personality, Attitudes, and Affect as Predictors of Second Language Communication", J. Language and Social Psychology, Vol. 15, pp. 3-26 (1996).

(16) Wit.ai, https://wit.ai/ (accessed 2015.5.1).

(17) AT\&T-TTS, http://www2.research.att.com/ ttsweb/tts/ (accessed 2015.5.1).

(18) CrazyTalk, http://www.reallusion.com/crazytalk/crazytalk. aspx (accessed 2015.5.1)

(19) Matsuoka, R.: "Willingness to Communicate in English among Japanese College Students", Proc. of the $9^{\text {th }}$ Conference of Pan-Pacific Association of Applied Linguistics, pp. 165-176 (2006).

(20) Sick, J. and Nagasaka, J. P.: "A Test of Your Willingness to Communicate in English (Japanese version)" (Unpublished Questionnaire) (2000).

(21) Yashima, T.: "International Posture and the Ideal L2 Self in the Japanese EFL Context", in Motivation, Language Identity and the L2 Self, eds. Dörnyei, Z. and Ushioda, E., pp. 144-163. Multilingual Matters, Clevedon, UK (2009). 


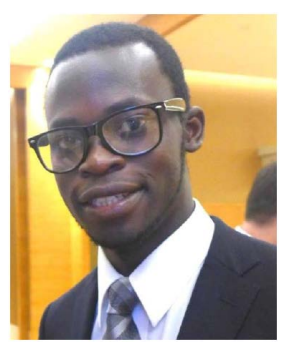

Emmanuel Ayedoun received his Bachelor's degree in Informatics from Osaka Prefecture University in 2016 and is currently doing a Master's in the same university. He received the Best Poster Presentation Award of the $11^{\text {th }}$ International Conference on Knowledge Management (ICKM 2015). His research interests include second language acquisition, embodied conversational agents and humancomputer interaction.

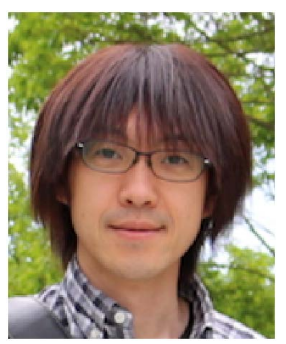

Yuki Hayashi received his Ph.D. degree in Information science from Nagoya University in 2012. From 2009 to 2012, he was a recipient of the JSPS research fellowship for young scientists (DCI). From 2012 to 2014 , he was an assistant professor in Seikei University. $\mathrm{He}$ is currently an assistant professor in the College of Sustainable System

Sciences and the Graduate School of Humanities and Sustainable System Sciences, Osaka Prefecture University. His research interests include computer-supported collaborative learning and human-computer interaction. He is a member of IPSJ, JSAI, JSiSE, and HIS.

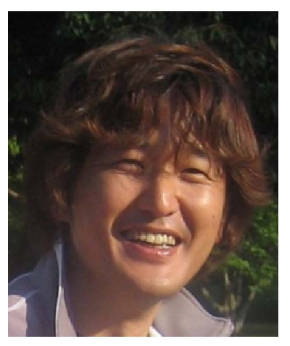

Kazuhisa Seta received the B.E. and M.E. from Ryukoku University in 1993 and 1995, respectively. He received the Ph.D. from Osaka University in 1998. He is currently a professor in the College of Sustainable System Sciences and the Graduate School of Humanities and Sustainable System Sciences, Osaka Prefecture University. His research interests include software engineering, intelligent tutoring systems, human resource management, and ontological engineering. He received Best Paper Awards of the Japanese Society for Information and Systems in Education in 2012 and 2015. He is a member of JSAI, IEICE, IPSJ, JSiSE, JCSS, and IAIED. 